\title{
Reversed-phase separation methods for glycan analysis
}

\author{
Gerda C. M. Vreeker ${ }^{1,2} \cdot$ Manfred Wuhrer $^{1,2}$ \\ Received: 30 May 2016 / Revised: 26 October 2016 / Accepted: 31 October 2016 / Published online: 25 November 2016 \\ (C) The Author(s) 2016. This article is published with open access at Springerlink.com
}

\begin{abstract}
Reversed-phase chromatography is a method that is often used for glycan separation. For this, glycans are often derivatized with a hydrophobic tag to achieve retention on hydrophobic stationary phases. The separation and elution order of glycans in reversed-phase chromatography is highly dependent on the hydrophobicity of the tag and the contribution of the glycan itself to the retention. The contribution of the different monosaccharides to the retention strongly depends on the position and linkage, and isomer separation may be achieved. The influence of sialic acids and fucoses on the retention of glycans is still incompletely understood and deserves further study. Analysis of complex samples may come with incomplete separation of glycan species, thereby complicating reversed-phase chromatography with fluorescence or UV detection, whereas coupling with mass spectrometry detection allows the resolution of complex mixtures. Depending on the column properties, eluents, and run time, separation of isomeric and isobaric structures can be accomplished with reversed-phase chromatography. Alternatively, porous graphitized carbon chromatography and hydrophilic interaction liquid chromatography are also able to separate isomeric and isobaric structures, generally without the necessity of glycan labeling. Hydrophilic interaction liquid chromatography, porous graphitized carbon chromatography, and
\end{abstract}

Published in the topical collection Glycomics, Glycoproteomics and Allied Topics with guest editors Yehia Mechref and David Muddiman.

Manfred Wuhrer

m.wuhrer@lumc.nl

1 Center for Proteomics and Metabolomics, Leiden University Medical Center, PO Box 9600, 2300 RC Leiden, The Netherlands

2 Division of Bioanalytical Chemistry, VU University Amsterdam, Faculty of Sciences, De Boelelaan 1083, 1081

HV Amsterdam, The Netherlands reversed-phase chromatography all serve different research purposes and thus can be used for different research questions. A great advantage of reversed-phase chromatography is its broad distribution as it is used in virtually every bioanalytical research laboratory, making it an attracting platform for glycan analysis.

Keywords Glycan · Reversed phase $\cdot$ Liquid chromatography $\cdot$ Separation

\section{Introduction}

Glycosylation is a frequently observed posttranslational modification in proteins. Many membrane and secretory proteins are glycosylated while passing through the endoplasmic reticulum and Golgi system [1]. Glycans are composed of monosaccharides that contain many chiral centers and are connected by glycosidic linkages. They may have very complex threedimensional structures [2], and stereoisomerism can have a substantial influence on the function of these molecules [3]. Various glycans are found in human cells; for example, $\mathrm{N}$ glycans or $O$-glycans that are linked to proteins, next to lipid-linked glycans and free molecules [4]. Structural and conformational differences in proteins can be caused by glycans, which may result in modulated protein activity and protein interactions $[3,5,6]$. These molecules participate in many different biological processes, such as cell signaling and recognition, immune defense, and parasitic infections [3].

The analysis of protein glycosylation can be performed on different levels - intact glycoproteins [7, 8], glycopeptides [9-12], and released glycans [13-15] — each resulting in slightly different information on the glycoprotein. A disadvantage of the analysis of intact glycoproteins is that good separation of the different glycoforms of a glycoprotein is hard to achieve, especially for proteins with many glycosylation sites 
and in complex samples [3]. The analysis of glycopeptides has the advantage that the glycosylation can be assigned to specific locations on the protein. This site-specific information can be used to assign specific glycan structures to distinct glycosylation sites. Furthermore, it can contribute to the understanding of the molecular structure of the protein $[9,16]$.

In this review the focus is on released glycans. Glycans can be released from proteins and peptides in an enzymatic and chemical way $[17,18]$. For $N$-glycans, various different enzymatic release methods are available, but for $O$-glycans, generally chemical release methods need to be used. The use of a chemical release method for glycans has several limitations: For example, the reducing-end aldehyde of the glycan can be reduced to an alditol by reductive $\beta$-elimination [19], thereby prohibiting subsequent labeling of the reducing end. After release, a derivatization step is often performed to improve the properties of the glycans for analysis. In addition, when one is working with complex biological samples, enrichment of the glycans needs to be performed. Hydrophilic interaction liquid chromatography (HILIC), graphitized carbon chromatography, and reversedphase solid-phase extraction are the most used methods for enrichment of glycans [20-26]. Besides these methods, methods based on graphene have been developed [27, 28].

Information on the released glycans can be gathered with several different techniques. Separation techniques such as capillary electrophoresis (CE) and liquid chromatography (LC) are often used in combination with mass spectrometry (MS), fluorescence, or UV detection. In addition, matrix-assisted laser desorption/ionization MS is used for glycan analysis without separation or in combination with LC fractionation. Also, gel electrophoresis is a commonly used technique for glycan analysis. Most of these techniques can be used for analysis of glycans in both their native form and their derivatized form.

For released glycan analysis, various LC stationary phases are used, including high-pH anion-exchange [29, 30], HILIC [31-33], porous graphitized carbon (PGC) [34-36], and reversed-phase stationary phases (see Table 1). In high-pH anion exchange, deprotonation of hydroxyl groups is achieved, which contributes to the separation of the glycans. Both native and derivatized glycans can be separated with this technique [29]. PGC separation is based on hydrophobic and polar interactions $[34,37]$. Native glycans are retained on the stationary phase and are eluted with water and acetonitrile [34]. Strong acidic or basic eluents can be used, because the columns are more hydrophobic and chemically stabler than reversed-phase columns [37].

The objective of this review is to compare reversedphase separation methods for glycan analysis. An overview of the literature on this subject is presented, with the emphasis on separation of the glycans investigated. Various labeling compounds are compared for their advantages in separation and detection. In addition, the elution orders of the glycans are discussed.

\section{Column specifications and configurations}

Reversed-phase chromatography is a widely used separation technique. An advantage of this technique is that it can be used in many laboratories, because only standard laboratory equipment is required [38]. In addition, various detection techniques can be used in combination with reversed-phase chromatography, depending on the labeling reagent used.

Reversed-phase separation is based on a noncovalent association between the nonpolar stationary phase and the nonpolar moieties of an analyte. The strength of this association depends on the polarity of the mobile phase [10]. The relative solubility of the analyte in the stationary phase and the mobile phase determines the degree of association of the analyte with the stationary phase and therefore the retention of the analyte. The retention is thus dependent on the competitive solubilization of the analyte between the stationary phase and the mobile phase.

An overview of the literature on reversed-phase separation of carbohydrates is presented in Table 1 . As can be seen, in almost all methods a $\mathrm{C}_{18}$ reversed-phase column is used for separation. Only two of the methods use a $\mathrm{C}_{8}$ column to separate analytes $[39,40]$. Although most methods are based on $\mathrm{C}_{18}$ separation, many different kinds of $\mathrm{C}_{18}$ columns are used. Reversed-phase chromatography is a commonly used analysis technique in chemistry and in other fields. Therefore an outstanding variety of $\mathrm{C}_{18}$ columns are commercially available. Columns with various different specifications are used. There are differences for example, in column length, internal diameter, and particle size, which may have a substantial influence on the separation efficiency. Differences between columns in terms of particle shape and bonded phase packing are illustrated by Snyder and Kirkland [41]. The hydrophobicity of the stationary phase also differs among columns [42]. In addition, the density and nature of the nonpolar groups immobilized on the silica surface will influence the selectivity [10].

Besides traditional and narrow-bore analytical reversedphase columns, the use of analytical nanoscale reversedphase columns is also described in several articles [38, 43-52]. Nano HPLC systems became commercially available in the 1990s. These nano HPLC columns typically have a dimension of $75 \mu \mathrm{m} \times 150 \mathrm{~mm}$ and a flow rate of around $300 \mathrm{~nL} / \mathrm{min}$. In addition, chip-based nano HPLC systems exist $[53,54]$. Unfortunately, reduction of the internal diameter of the column will also limit the amount of sample that can be injected. To facilitate larger injection volumes, trapping columns are used. The analytes are trapped on a small column with relatively high flow rates and often large injection volumes followed by elution onto the longer analytical column for separation $[55,56]$. By reduction of the internal diameter, the sensitivity of the measurements is increased with MS detection: sensitivities in the low femtomole range can be achieved in MS and MS/MS mode [53, 57, 58]. In addition, 


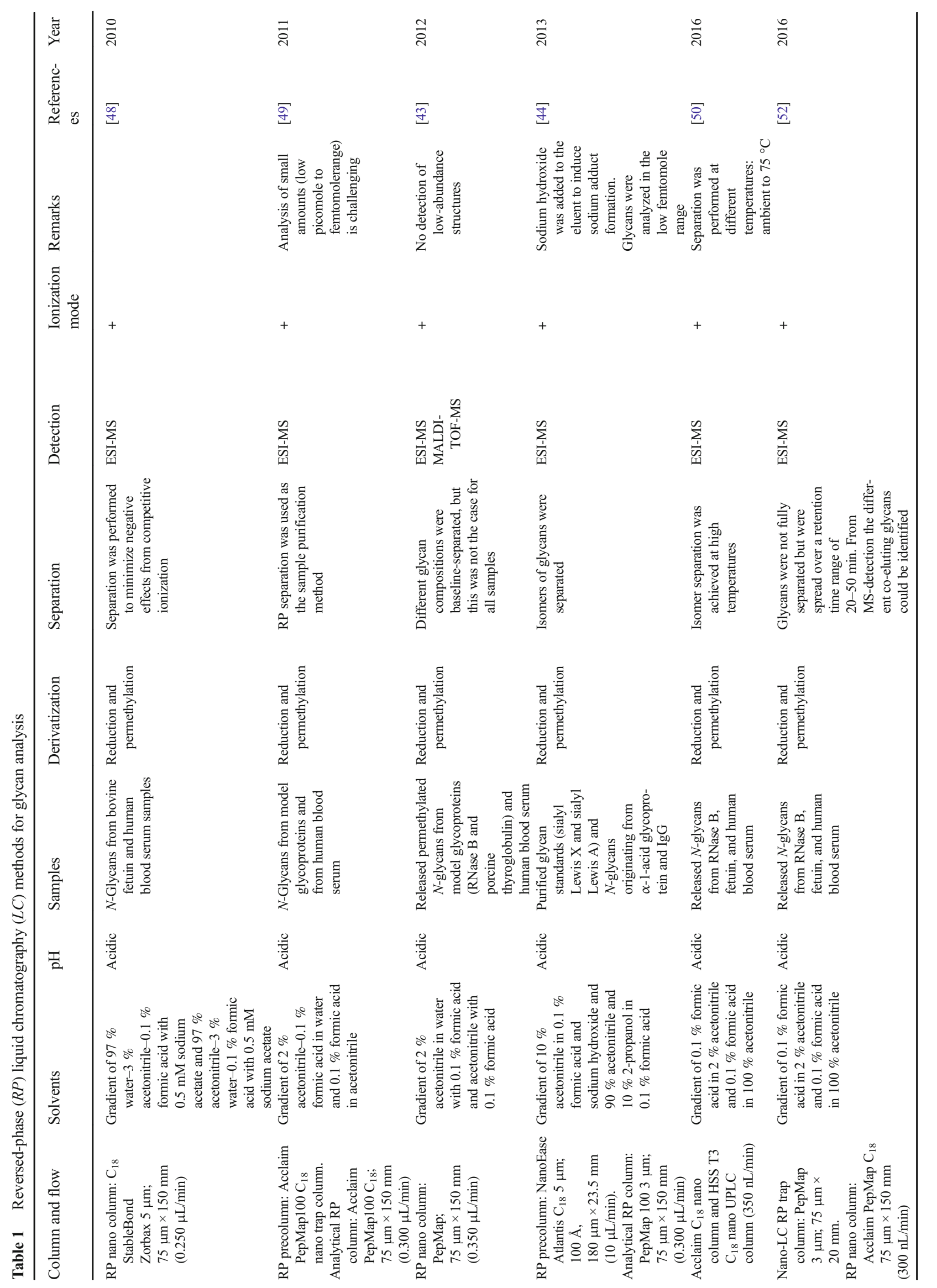




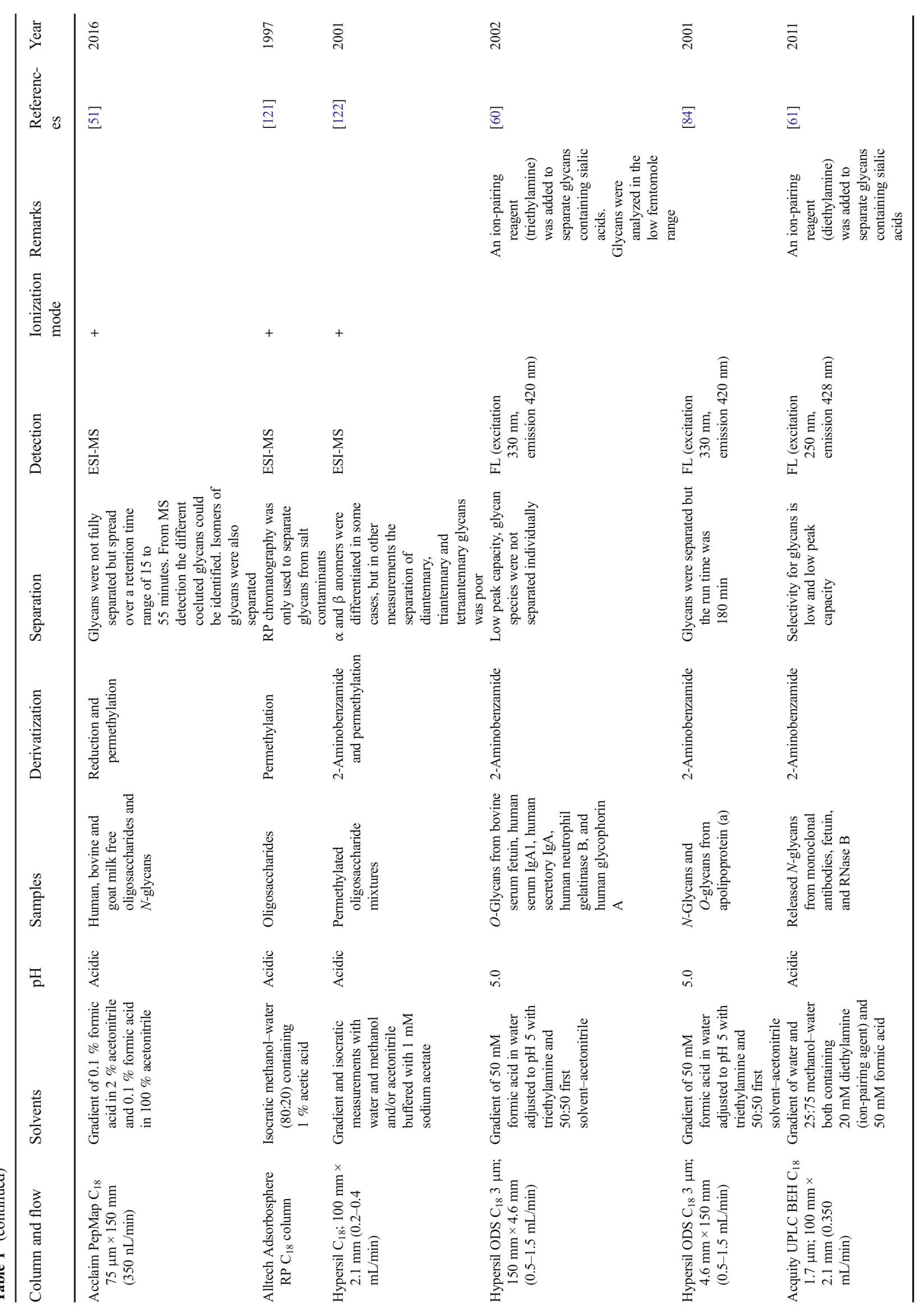




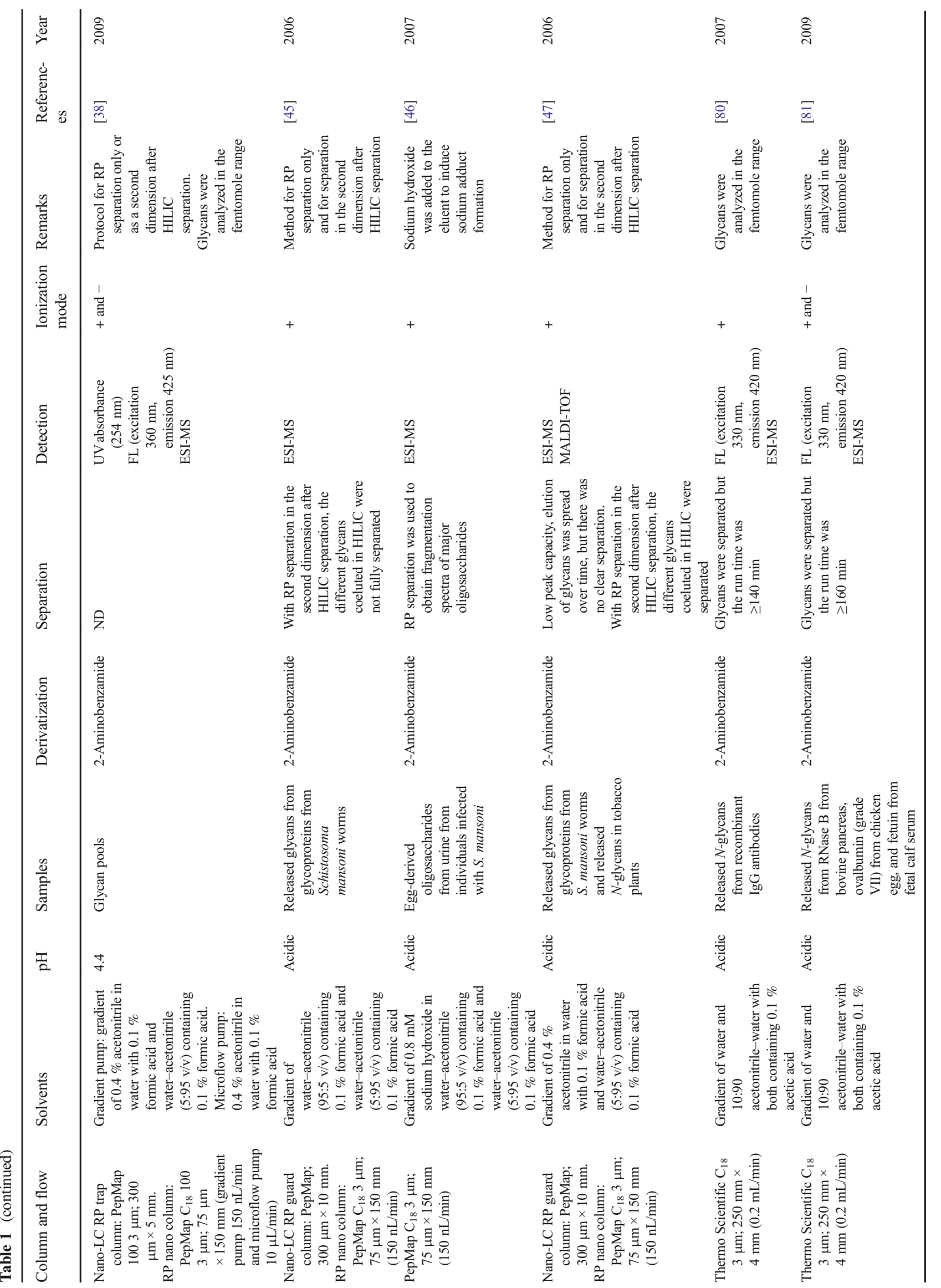




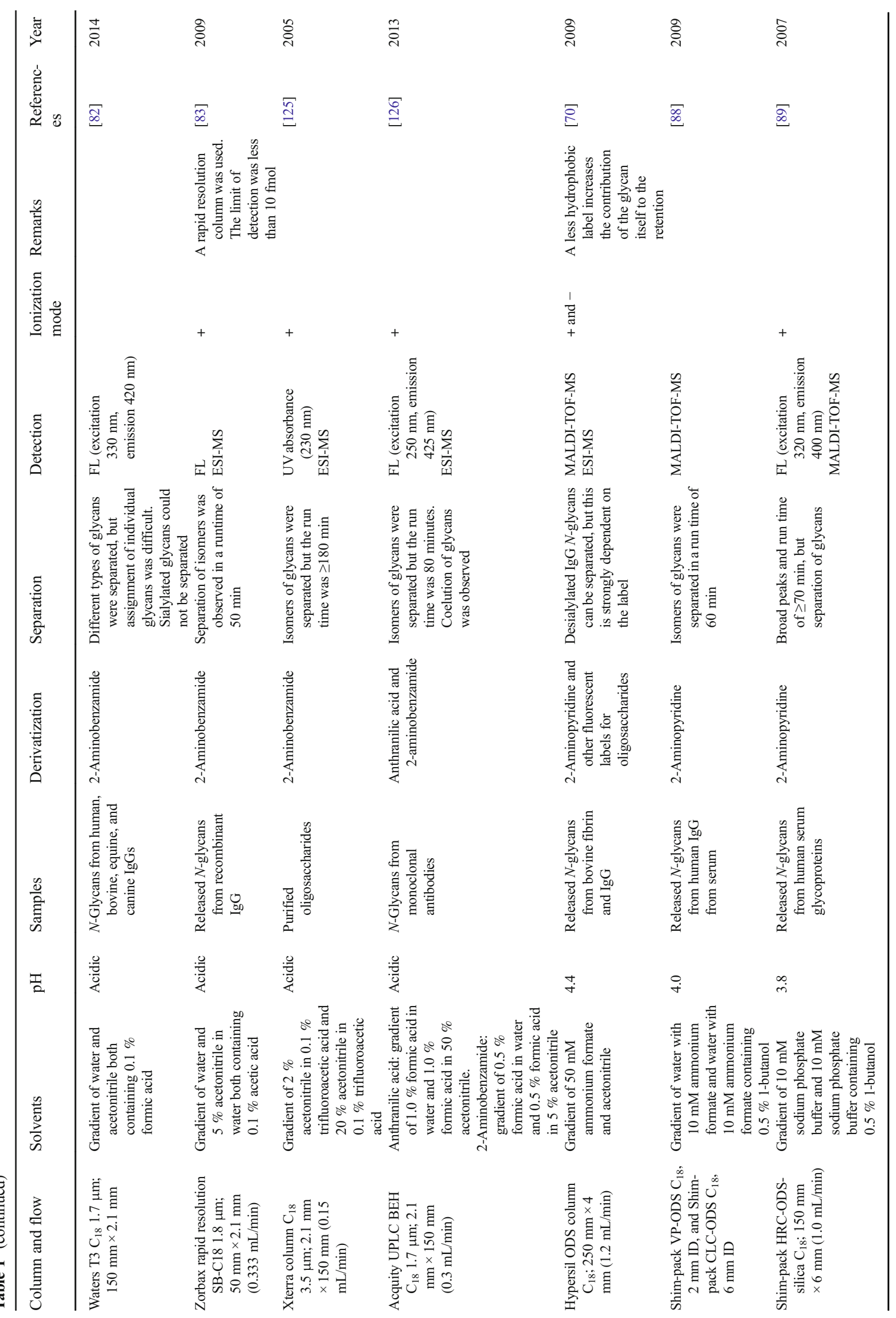




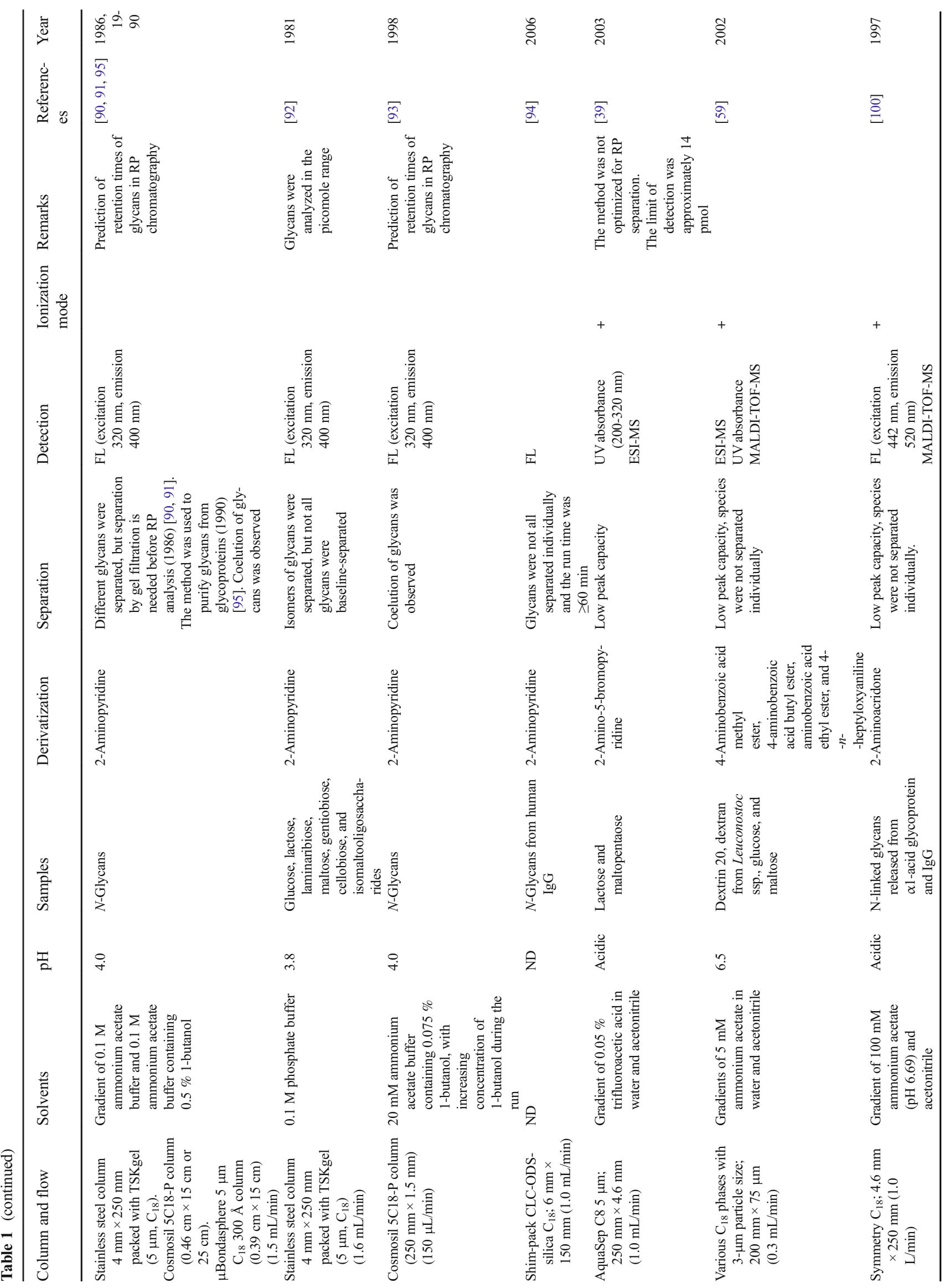




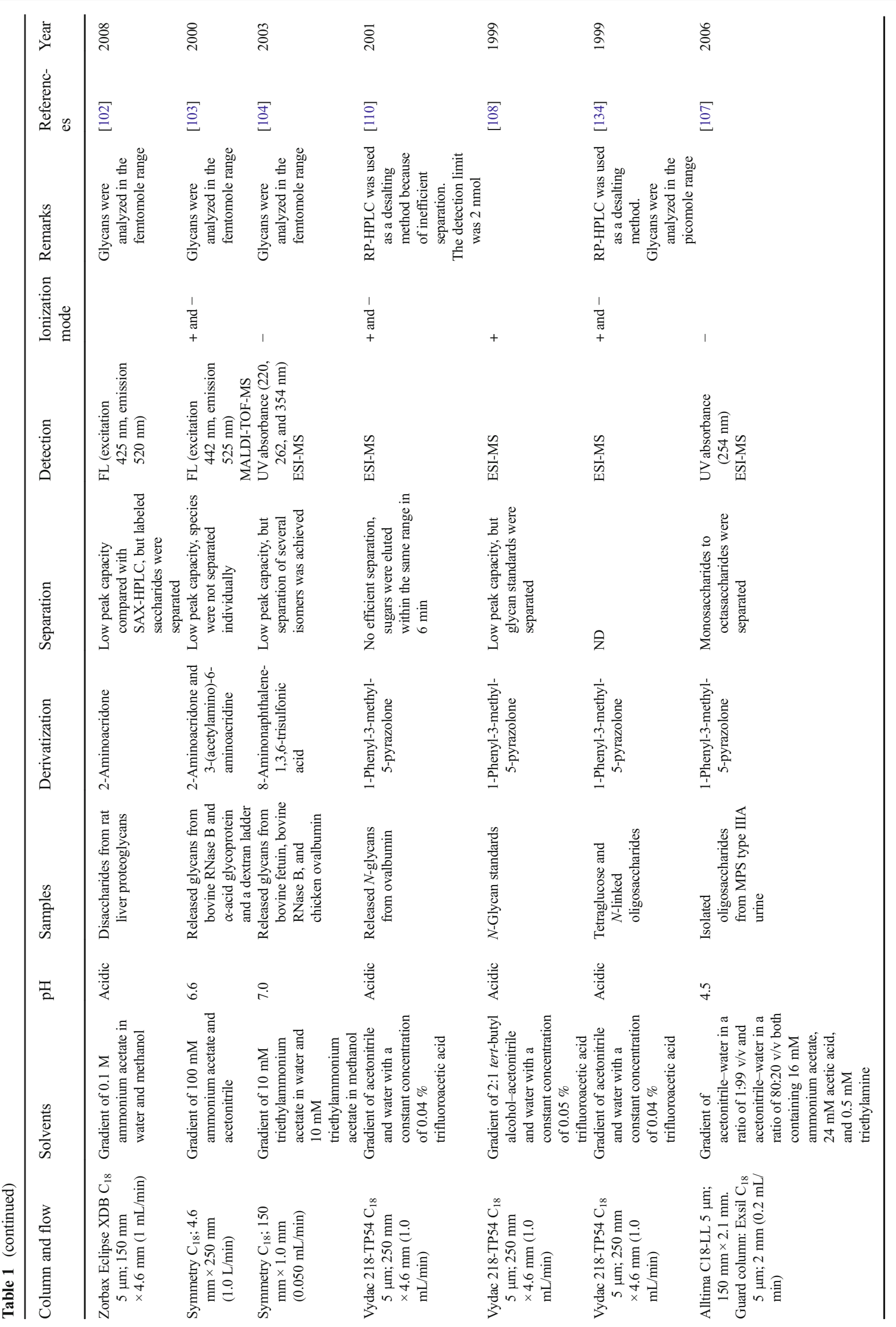




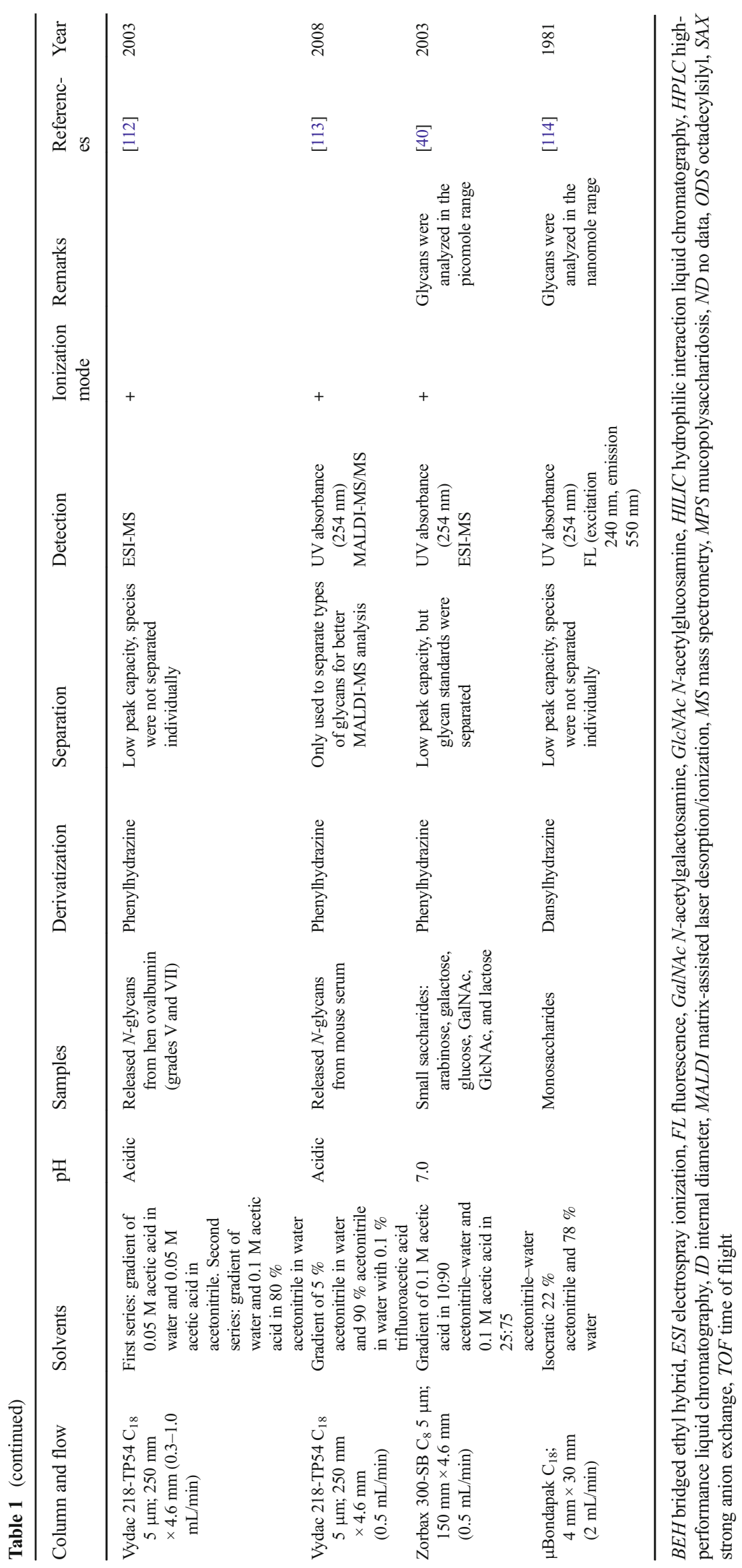


the use of nanoscale columns increases the separation efficiency and resolution, as was shown by Schmid et al. [59]. The coupling of nanoscale columns with MS detection is of importance for glycan analysis as the samples are often complex and full chromatographic separation is not obtained. Of note, separation of isobaric and isomeric glycan species is of particular value in combination with MS detection to achieve detailed characterization of complex glycan samples.

Full chromatographic separation, or at least the separation of isomers, can be achieved by use of two analytical columns. In work reported in a few articles, a reversed-phase column was used in the second dimension of two-dimensional LC [38, 45-47]. In the first dimension, separation was performed in HILIC mode at analytical scale, resulting in an incomplete separation of the different glycans in the samples. By use of nanoscale reversed phase in the second dimension, separation of complex samples and isomer mixtures was achieved. With this two-dimensional separation with nanoscale MS detection, detailed characterization of complex glycan mixtures can be achieved with high sensitivity.

\section{Solvents}

As can be seen from Table 1, both gradient and isocratic runs are performed. In all cases the $\mathrm{pH}$ of the solvents is acidic or neutral. Most methods use a binary gradient, with solvent A consisting of mainly water and solvent B consisting of acetonitrile, methanol, or a mixture of one of these solvents with water. Solvent A may also contain up to $10 \%$ acetonitrile. In addition, both solvents may contain low concentrations of a volatile acid (formic acid, acetic acid, or trifluoroacetic acid). Dependent on whether electrospray ionization (ESI) MS is used or not, volatile buffers (sodium acetate, triethylammonium acetate, ammonium acetate, or ammonium formate) or nonvolatile buffers (sodium phosphate) are used. In two cases, low amounts of sodium hydroxide were added to solvent $\mathrm{A}$ to induce sodium adduct formation in MS [44, 46]. In addition, in a few methods an ion-pairing reagent (diethylamine and triethylamine) is used to support retention of glycans with charged groups such as sialic acids $[60,61]$. Dependent on which label was used, some adjustments in the solvents were made.

\section{Derivatization and detection}

Although techniques that do not require derivatization exist [62], generally glycans are derivatized before reversed-phase separation and analysis. Carbohydrates absorb light only at low wavelengths, which results in low sensitivity in UV and fluorescence detection. In addition, amperometric and refractive index detection also have a problem with sensitivity when carbohydrates are analyzed. Coupling UV-absorbing and fluorescent molecules to these analytes greatly enhances the detection sensitivity in, for example, HPLC and CE [29]. Derivatization can also be used to increase detectability in MS by use of labels with a substituent that can be charged; for example, an amine or carboxylic acid group [29]. Also, derivatization with a hydrophobic label can increase sensitivity in ESI-MS, which was examined by Williams et al. [63]. However, a hydrophobic bias, which depends on the chemical and physical properties of the analytes, is present in ESI-MS [64]. In 1993, Fenn [65] described that ion desorption from droplets is dependent on the surface activity of the analyte. He compared this with the hydrophobicity of the analyte and showed in an example that the MS response increases with the number of carbon atoms per alkyl chain in tetraalkylammonium halides. This hydrophobic bias has consequences for quantitation by ESI-MS [66]. In addition, the signal intensity in ESI-MS depends on the amount of organic solvent present. For instance, the use of $80 \%$ acetonitrile as the solvent showed a sixfold increase in intensity in the study of Bleicher and Bayer [67]. Moreover, the total signal intensity per analyte is also decreased by their being multiple charge states [68]. Quantitation by MS detection may for these reasons be compromised, unless an internal standard with properties similar to those of the analyte (e.g., an isotopically labeled version of the analyte) is used for absolute quantitation [69].

To be able to analyze carbohydrates with reversed-phase chromatography, it is important to make the analytes more hydrophobic so they can interact with the alkyl chains in the stationary phase. As shown in Table 1, various labels are used in reversedphase chromatography of glycans: 2-aminobenzamide (AB), anthranilic acid (AA), 2-aminopyridine (PA), 2-amino-5bromopyridine (ABP), 4-aminobenzoic acid methyl ester (ABME), 4-aminobenzoic acid ethyl ester (ABEE), 4aminobenzoic acid butyl ester (ABBE), 4- $n$-heptyloxyaniline (HOA), 2-aminoacridone (AMAC), 3-(acetylamino)-6aminoacridine (AA-Ac), 2-aminonapthalene trisulfone (ANTS), 1-phenyl-3-methyl-5-pyrazolone (PMP), phenylhydrazine, dansylhydrazine, and the individuality normalization when labeling with isotopic glycan hydrazide tags (INLIGHT). The structures of these labels are shown in Fig. 1. Besides labeling of the reducing end, reduction of the reducing end and permethylation of the glycan were also performed (Table 1). Another reducingend label gaining popularity for HILIC analysis with fluorescence detection is procainamide [70,71], yet its use in reversephase chromatography has still to be established. Likewise, labels that target glycosylamines generated by PNGase $\mathrm{F}$ release of $N$-glycans such as Instant $\mathrm{AB}^{\mathrm{TM}}[72,73]$ may be suitable for reversed-phase separation of glycans yet these analyses have still to be developed. Recently isotopic aminoxyTMT labels were introduced by Afiuni-Zadeh et al. [74], and these can be used for quantitation of peptides as well as glycans in MS. Zhou et al. [75] used these labels for glycan analysis on a PGC column, and stated that the label was not hydrophobic enough to obtain effective separation on a reversed-phase column. 
Fig. 1 Structures of labels used in reversed-phase chromatography of oligosaccharides. $A A$ anthranilic acid, $A A-A c$ 3-(acetylamino)-6aminoacridine, $A B$

2-aminobenzamide, $A B B E$

4-aminobenzoic acid butyl ester, $A B E E$ 4-aminobenzoic acid ethyl ester, $A B M E>4$-aminobenzoic acid methyl ester, $A B P$ 2-amino-5bromopyridine, $A M A C$

2-aminoacridone, ANTS

2-aminonapthalene trisulfone,

HOA 4- $n$-heptyloxyaniline,

INLIGHT individuality normalization when labeling with isotopic glycan hydrazide tags, $P A$ 2-aminopyridine, $P M P$ 1-phenyl-3-methyl-5-pyrazolone<smiles>NC(=O)c1ccccc1N</smiles>

AB<smiles>[R]OC(=O)c1ccc(N)cc1</smiles>

ABME, $R=$ methyl ABEE, $R=$ ethyl<smiles>Nc1cc(S(=O)(=O)[O-])cc2cc(S(=O)(=O)[O-])cc(S(=O)(=O)[O-])c12</smiles>

ANTS ABBE, $R=$ butyl<smiles>Nc1ccccc1C(=O)O</smiles>

AA<smiles>Nc1ccccn1</smiles>

PA<smiles>Nc1ccc(Br)cn1</smiles>

$\mathrm{ABP}$<smiles>CCCCCCCOc1ccc(N)cc1</smiles><smiles>CC1=NN(c2ccccc2)C(=O)C1</smiles>
PMP<smiles>CC(=O)Nc1ccc2cc3ccc(N)cc3nc2c1</smiles>

AA-AC<smiles>NNc1ccccc1</smiles>

phenylhydrazine<smiles>CN(C)c1cccc2c(S(=O)(=O)NN)cccc12</smiles>

dansylhydrazine<smiles>NNC(=O)Cc1ccc(CCc2ccccc2)cc1</smiles>

INLIGHT
Most labels described in Fig. 1 can be coupled to glycans by reductive amination, which is the most commonly used glycan derivatization technique. In this reaction, first a Schiff-base intermediate is formed. This Schiff base is subsequently reduced with sodium cyanoborohydride or 2-picoline borane to form a stable secondary amine $[76,77]$. This derivatization reaction is often performed in methanol or dimethyl sulfoxide with acetic acid added to the organic solvent [78]. An important feature of this method is the stoichiometric coupling of one label per glycan, which together with the usually high labeling efficacies makes quantitation by fluorescence or UV detection possible [29]. The reaction mechanism for Schiff-base formation was described by Anumula [79], who showed that the labeling reaction is initiated by the attack of the lone pair of the amino group of the label on the carbon of the aldehyde of the reducing end of the glycan.

Various molecules containing an amino group can be coupled to glycans by reductive amination. The most commonly used label for glycan analysis is AB, which is used for the analysis of $N$-glycans [61, 80-84] as well as $O$-glycans $[60,84]$. This label can be combined with various glycoanalytical methods, including several chromatographic phases (e.g., HILIC and reversed phase) and detection methods (e.g. UV detectors, fluorescence, and MS) $[85,86]$. AA is a label that is similar to AB. This label contains a carboxylic acid moiety instead of the amide group. Labeling with AA provides at least two times more sensitivity in fluorescence detection as compared with labeling with $\mathrm{AB}$ [79]. In addition, $\mathrm{AB}$ and AA can both be used in positive and negative ion mode MS [32, 38, 81]. The labeling procedures for AA and AB labels were optimized by Bigge et al. [87] in the 1990s. They measured a labeling efficiency of more than $80 \%$ for $\mathrm{AB}$ and approximately $80 \%$ for AA. These labeling efficiencies were also investigated by Ruhaak et al. [77], who reported that, with sodium cyanoborohydride and 2picoline borane as reducing agents, in most cases an almost complete labeling of glucose polymers was obtained.

Another label that is often used for labeling at the reducing end by reductive amination is PA [70, 88-95]. Hase et al. [92] were the first to use PA labeling for HPLC analysis of glycans. PA has a relatively low hydrophobicity compared with the other labels mentioned, which results in a rapid desorption from the stationary phase, providing sharp elution peaks [96]. In many of the methods using PA, a buffer was added to the solvents to maintain a low $\mathrm{pH}$. As the $\mathrm{p} K_{\mathrm{a}}$ of PA is 6.8, the molecule is protonated at low $\mathrm{pH}$ [97]. Testa and Wild [98] showed that this protonation results in increased fluorescence. This is beneficial for fluorescence detection, which is often used in combination with this label (Table 1). As a consequence of the low hydrophobicity of the label, low concentrations of organic solvents are used in the eluents, 
which makes direct coupling with ESI-MS less convenient [70]. ABP is PA with an additional bromine moiety. This bromine is advantageous when MS analysis is used, because of the natural equal abundances of ${ }^{79} \mathrm{Br}$ and ${ }^{81} \mathrm{Br}$. In high-resolution MS, signals resulting from labeled species can easily be recognized by the typical isotopic pattern [39].

ABME, ABEE, ABBE, and HOA are less commonly used glycan derivatization reagents. These labels were compared by Schmid et al. [59], who showed that the retention times increased with a longer alkyl chain at the 4-position of the label. The polarities of the glycans tend to be very similar after derivatization with ABME, resulting in rapid elution and poor resolution for large glycans. Longer retention and better separation were observed for the other three labels [59, 99]. Sensitivity increases for positive and negative mode ESI-MS were tested by Pabst et al. [70], who showed that in positive mode, ABEE increased sensitivity by a factor of 2 compared with the native glycans. In negative mode a slight increase in sensitivity was observed with ABEE and ABBE.

$A M A C$ is a labeling reagent with pronounced hydrophobic properties [100]. The molecule has fluorescent properties and a strong UV absorbance, which can be used for fluorescence analysis [101, 102]. Nevertheless, the fluorescence sensitivity of AMAC was somewhat less than that of $\mathrm{AB}$ and around four times than that of AA [32]. Charlwood et al. [103] reported AA-Ac based on AMAC as a new derivatization reagent. This label has higher fluorescence intensity than AMAC [103]. In positive ion mode ESI-MS, an intensity gain of a factor 2 was observed for AA-Ac compared with AA, whereas for AMAC the intensity was considerably lower [70].

ANTS is a charged molecule, which means that the label is hardly retained in reversed-phase chromatography [104]. Although it might not seem a natural choice to use this charged label in reversed-phase chromatography, Gennaro et al. [104] used it to separate glycans with a sensitivity in the low femtomole range. An ion-pairing reagent, triethylammonium acetate, was added to the mobile phase to create neutral complexes with the label, which then could be retained by the reversed-phase stationary phase. Like ANTS, 8-aminopyrene-1,3,6-trisulfonic acid, which is similar to ANTS, has been used for glycan separation on a reversephase stationary phase [105]. Glycans derivatized with ANTS were reported to have increased sensitivity in ESIMS as compared with native glycans [104]. However, this increase was not observed by Pabst et al. [70].

ANTS was first used as a fluorophore in gel electrophoresis. In addition, it is used as a derivatizing reagent for $\mathrm{CE}$ and HILIC separations [29, 106]. Analysis of ANTS derivatives by reversed-phase chromatography can provide information on isomers and can be used as a technique complementary to CE and HILIC [104]. Reversed-phase separation of ANTS-derivatized glycans was obtained with an ion-pairing reagent. Without this ion-pairing reagent, ANTS-labeled analytes are found in the flow-through [104]. Various labels coupled to the reducing end by reductive amination were compared by Pabst et al. [70]. Unfortunately the derivatization protocols were not optimized, and thus no labeling efficiencies were shown.

PMP is a label that is added with a stoichiometry of two labels per glycan [107]. This addition of two labels might be a disadvantage, as it leads to a rather bulky reducing-end modification that may dominate separation. The labeling reaction with PMP is a Michael addition, which is performed under alkaline conditions [29]. In these alkaline conditions, loss of the sialic acids during derivatization is prevented [108]. PMP is a UV-absorbing molecule, but does not have fluorescent properties [29, 78]. In positive ion mode in ESI-MS, the sensitivity is approximately double that of native glycans, whereas in negative mode this gain was not observed [70]. PMP has been used for CE, HILIC and reversed-phase chromatography separations of glycans [109-111]. Saba et al. [110] observed in reversed-phase chromatography an elution range of only 6 min for the $N$-glycans of ovalbumin, whereas for HILIC the glycans were eluted over a range of $35 \mathrm{~min}$. In addition, in-source fragments were observed in reversed phase LC-MS, whereas these were not observed with HILIC coupling. For these reasons, it was concluded that reversed-phase conditions were more useful for desalting PMP-derivatized samples than as a separation method [110, 112].

Derivatization of glycans can also be performed with hydrazine labels [40, 112-114]. The carbonyl group of the reducing-end aldehyde reacts with the hydrazine moiety to form a hydrazone bond [78]. This reaction is relatively clean, because no salts are used or produced during the reaction, which makes sample cleanup after the reaction often not necessary [40]. High labeling efficiencies for phenylhydrazines were observed for this reaction by Lattova and Perrault [40]. Dependent on the analysis technique, various hydrazinecontaining molecules can be coupled to glycans; for example, dansylhydrazine, which has fluorescent properties and thus can be used in fluorescence detection [114]. Another hydrazine label for glycan analysis, the INLIGHT label, was introduced by Walker et al. [115] in 2011. There are two variants of this label, one of which contains six ${ }^{13} \mathrm{C}$ atoms and the other one does not. The use of both labels makes quantification by MS possible. These labels can be used in combination with HILIC separation [116, 117], but reversed-phase separations are also performed $[118,119]$. Unfortunately, no information was given on the separation efficiency of glycans with these labels.

In native glycans, the monosaccharide at the reducing-end terminus equilibrates between an open-ring conformation and a closed-ring conformation [2]. In the closed-ring conformation the $\mathrm{C}-1$ atom is a chiral center with $\alpha$ and $\beta$ anomers. In chromatography, small differences or double peaks could be observed for saccharides with a degree of polymerization of 
three monomers or more, because of these $\alpha$ and $\beta$ anomers in the molecules. To overcome this without further labeling of the glycan, the reducing-end aldehyde in the open-ring conformation can be reduced with a reducing agent such as sodium borohydride, to form an alditol [29, 44, 120]. Other methods to eliminate double peaks (e.g., increasing the column temperature) exist but they will not be discussed in this review [10].

Glycans can also be derivatized by permethylation [43, $44,48,121,122]$. In contrast to the other derivatization methods discussed in this review, this derivatization does not occur at the reducing end. The hydrogens on the oxygen and nitrogen atoms in the glycans are replaced by methyl moieties, resulting in a largely hydrophobic molecule [122, 123]. This change in polarity and hydrophobicity is beneficial for glycan analysis with reversed-phase chromatography. In addition, permethylated compounds show an increase in detection sensitivity in ESI-MS, but detection in the femtomole range is challenging $[48,49,122]$. Notably, sialic acids are stabilized by permethylation via esterification, thereby preventing the ready loss of sialic acids observed in matrix-assisted laser desorption/ionization time-of-flight MS analysis of native glycans [124].

\section{The influence of reducing-end labels on glycan retention}

Glycans are hydrophilic molecules that tend to be hardly retained on many reversed-phase materials. Various labeling techniques may help to achieve glycan retention reversedphase separation. An overview of the retention of native glycans as well as some labeled glycans is given in Fig. 2a. PA is the least hydrophobic of the commonly used labels and thus has a relatively low interaction with the stationary phase. The weak interactions of this label with the stationary phase results in the contribution of the glycan itself to the retention being relatively high with the PA tag compared with more hydrophobic tags [70]; therefore structural information can be derived from the glycans derivatized with this label. With the small, low-hydrophobicity PA label, specific glycan structural details tend to have relatively large influences on separation, which results in better separation of different species, as was shown by Pabst et al. [70]. They compared the separation of PA-, AB- and ABEE-labeled immunoglobulin $G$ glycans, which is shown in Fig. $2 b$ and c. In the case of PA-labeled glycans, small complex glycans were eluted first, whereas for ABEE-labeled glycans, the larger complex glycans were eluted first. This can be explained by the aforementioned contribution of glycans to the retention. In the case of PA, the retention is for a large part dependent on the interaction of the glycan with the stationary phase. Thus if the glycan has a higher degree of polymerization, it may engage in more interactions, resulting in longer retention. In the case of ABEE, the retention is mainly based on the hydrophobic label and the size of the hydrophilic glycan. One may speculate that the glycan interferes with the interaction of ABEE and the stationary phase, thereby leading to earlier elution of glycans with increasing degree of polymerization. For AB-labeled glycans, the glycan hardly modulates the retention contribution of the tag, resulting in a relatively poor reversed-phase separation of glycans [70]. Nevertheless, the separation of AB-labeled glycans can still be accomplished, with use of long gradients

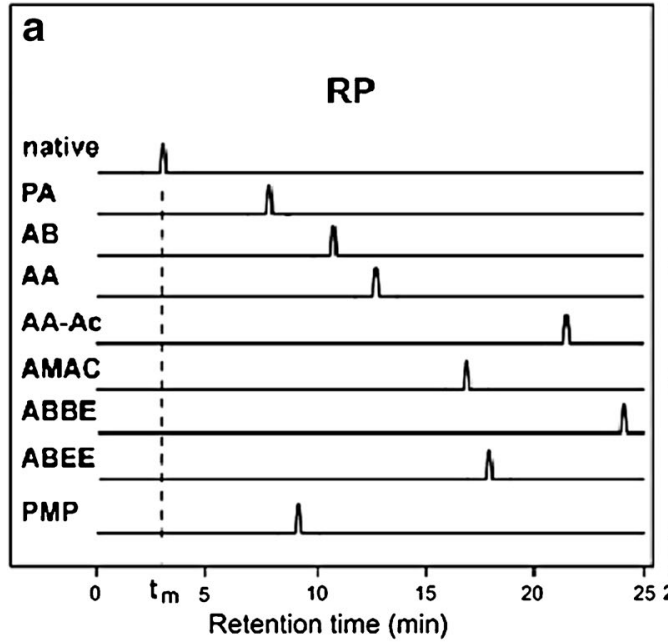

Fig. 2 a Overview of the chromatograms of various reducing end derivatized $N$-glycans and a native glycan that is eluted at the void volume (dashed line). b Reversed-phase chromatograms of desialylated immunoglobulin $\mathrm{G} N$-glycans derivatized with $\mathrm{PA}, \mathrm{AB}$, or ABEE. c Chromatograms of the separation of a mixture of four glycans:

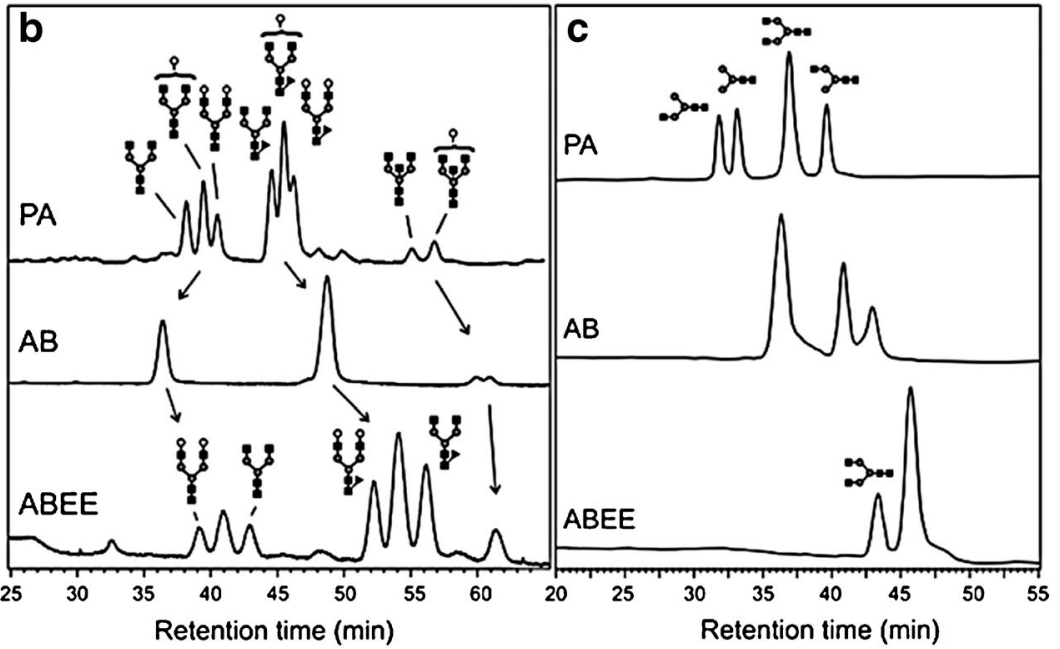

$(\mathrm{GlcNAc})_{2}(\mathrm{Man})_{3}(\mathrm{GlcNAc})_{2},(\mathrm{GlcNAc})_{2}(\mathrm{Man})_{3}$, and two isomers of $(\mathrm{GlcNAc})_{2}(\mathrm{Man})_{3}(\mathrm{GlcNAc})$, where GlcNAc is $N$-acetylglucosamine and Man is mannose. $R P$ reversed phase. (Reproduced and modified from Pabst et al. [70] with permission) 
$[80,84,125]$. In this separation a negative correlation between the retention and the size of the high-mannose glycans was observed, whereas for complex glycans this correlation was positive $[61,126]$.

Schmid et al. [59] investigated on the separation of glycans labeled with ABME, ABEE, ABBE, and HOA. The first three labels mentioned differ by the length of the alkyl chain at the para position from the amine, and HOA contains an $n$ heptoxyl group at the para position. It was shown that this small difference in chain length had a substantial influence on the separation of the glycans and the run time per sample. The retention times are longer for $\mathrm{ABEE}$ and $\mathrm{ABBE}$ than for ABME because these labels are more hydrophobic. The longest retention times were measured for HOA. Better separations, including baseline-separated peaks, were obtained for ABEE, ABBE, and HOA.

Besides the different labels, Schmid et al. also tested different $\mathrm{C}_{18}$ stationary phases (i.e., differences in end-capping, pore size, etc.) for the separation of ABBE-labeled glycans, and showed differences in separation and retention times. One of these stationary phases was, for example, coated with a reactive polymeric silicone film that chemically binds to the silica gel and is alkylated afterward. For this encapsulated stationary phase, baseline separation of most of the analytes was observed, whereas for the partially trimethylsilyl endcapped stationary phase, overlap between all analytes was observed [59]. Furthermore, Gillmeister et al. [88] compared columns from various manufacturers, and showed major differences in retention and separation of glycans. The choice of the $\mathrm{C}_{18}$ column for separation can thus have a substantial influence on the separation efficiency.

\section{Separation of permethylated glycans}

Permethylation of glycans has also regularly been used as a derivatization technique followed by reversed-phase LC-MS (Table 1). In this case the glycan itself is made less hydrophilic by the substitution of hydrogen atoms for methyl groups. This separation is thus based on the properties of the glycan and results in the smallest glycans being eluted first. Coelution of permethylated glycans is observed for the more complex samples, but because of MS detection these overlapping glycan species can still be identified [43, 51, 52]. Ritamo et al. [44] and Zhou et al. [50] observed separation of isomers, although in some cases the sample complexity was limited. The latter researchers performed the separation of permethylated glycans at higher temperature, which increased the chromatographic resolution of isomers and improved the peak shape and decreased the peak width. In addition, it was concluded that the influence of the three-dimensional structure of the glycans was reduced because of the higher-temperature separations, resulting in more predictable retention times, which could be beneficial for the identification of glycans.

\section{Glycan structural features influencing retention}

AB labeling is often used in LC-MS methods. The separation of glycans labeled with $\mathrm{AB}$ was studied by Prater et al. [83] and Higel et al. [126], who showed that oligomannose glycans are eluted first. The main separation of the complex and hybrid glycans is caused by the core fucose: glycans containing a core fucose are eluted later than glycans without core fucosylation. Within these two groups, the acidic hybrid and complex glycans are eluted before the neutral hybrid and complex glycans, with the hybrid glycans being eluted before the complex ones. For AA labeling this elution order is almost the same [126]. Unfortunately, no information on isomers, bisecting variants, or triantennary variants was presented. Notably, when an ion-pairing reagent is used in combination with AB-labeled glycans the retention time of the sialylated glycans increases, which results in elution of sialylated glycans after neutral glycans [61]. Chen and Flynn [80] also performed reversed-phase separation of AB-labeled $N$-glycans, and found a slightly different elution order of the different types of glycans. They described that fucosylated sialylated glycans are eluted first from the column, followed by high-mannose glycans and neutral complex glycans [80, 81]. The difference between the elution order described by Higel et al. and this elution order might be due to differences in the columns, eluents, and gradients used, but this was not investigated. In addition Chen and Flynn [80] also described the elution of fucosylated triantennary and tetraantennary glycans. With their method these triantennary and tetraantennary glycans are eluted after the nonfucosylated glycans and before fucosylated diantennary species.

PA-labeled glycans show an increased relative retention of glycans containing a higher number of sialic acids [127, 128]. In contrast, for PMP-derivatized glycans the sialylated glycans are eluted earlier than $(\mathrm{GlcNAc})_{2}(\mathrm{Man})_{3}$, where GlcNAc is $N$ acetylglucosamine and Man is mannose, but in this case it cannot be said if this was due to the size of the glycans or the sialic acids present [108]. Tomiya and Takahashi [128] determined the elution positions of various neutral and sialylated PA-derivatized glycans. They calculated the contributions of the different monosaccharides at various positions in the $N$ glycan, taking linkages into account. These contributions were expressed in glucose units, which can be calculated by comparison of the retention times of the glycans with the retention times of the standard PA-isomaltooligosaccharides or a standard dextran ladder. The advantage of using glucose units instead of retention times is that glucose units are largely independent of the system and column used, which makes glucose units highly reproducible [60, 128]. 
The contribution of sialic acids to the retention on a $\mathrm{C}_{18}$ stationary phase strongly depends on the linkage (e.g. $\alpha 2,3$ linkage vs $\alpha 2,6$ linkage) and also on the type of sialic acid present (e.g., $\mathrm{N}$-acetylneuraminic acid or $\mathrm{N}$-glycolylneuraminic acid). In addition, the retention is also highly dependent on which antenna is sialylated [128]. For glycopeptides the elution order of neutral and sialylated species is the opposite: neutral glycan chains are eluted earlier than sialylated glycans with the same peptide moiety. Glycopeptides containing two sialic acid moieties on their glycans are eluted even later [129]. It appears that the different contributions of sialic acids to the retention of reducing-end labeled glycans as well as glycopeptides is still incompletely understood.

Besides the presence of sialic acids, also bisection of the glycan can influence the retention on the reversed-phase stationary phase. The addition of a bisecting GlcNAc to PAlabeled glycans results in a strong positive contribution to the retention [128]. The retention time increases even more if the bisecting GlcNAc contains a $\beta 1$,4-linked galactose, which is a glycan structure that was discovered in immunoglobulin $\mathrm{G}$ by Takegawa et al. [130].

The separation of AMAC-labeled glycans was described by Okafo et al. [100]. The elution order of glycans was comparable to the elution order of the other relatively more hydrophobic labels, thus with the tetraantennary glycans being eluted before the triantennary glycans, which are eluted before the diantennary glycans. Okafo et al. [100] also found that antenna fucosylated glycans were eluted earlier than their nonfucosylated analogues, whereas the core-fucosylated glycans showed more retention than their nonfucosylated analogues.

This elution order of fucosylated glycans was observed not only for AMAC-labeled glycans but also for AB- and PAlabeled glycans [47, 128]. Tomiya and Takahashi [128] showed with their calculations on PA-labeled glycans that a core fucose on a $\mathrm{N}$-glycan can have a major effect on the retention of the glycan on a reversed-phase stationary phase. This effect again depends on the linkage of this fucose (e.g., $\alpha 1,6$-linked fucose or $\alpha 1,3$-linked fucose). In vertebrate $N$ glycans, mainly $\alpha 1,6$-linked core fucoses are present, and these fucoses have a large positive contribution to the retention on a $\mathrm{C}_{18}$ stationary phase. Furthermore, the contribution of antenna fucosylation was also calculated. This contribution again depends on on which antenna the fucose is located and the linkage to this antenna. It was calculated that all antenna fucoses have no or a negative contribution to the retention in a $\mathrm{C}_{18}$ stationary phase [128]. This difference in retention of core and antenna fucoses might be explained by the polarity of a fucose. Fucoses are relatively apolar monosaccharides compared with the other monosaccharides present in $\mathrm{N}$-glycans, because of their methyl group. In core-fucosylated glycans, the fucose is located relatively close to the label that interacts with the stationary phase, and both may interact concertedly with the stationary phase, leading to increased retention. In contrast, when the fucose is positioned on one of the antennae such a concerted interaction is not likely, which may explain the slightly lower retention of antenna-fucosylated glycans as compared with core-fucosylated glycans [47, 100, 128]. In PGC analysis of fucosylated glycans it was similarly found that antenna-fucosylated glycans are eluted before corefucosylated glycans [131]. The different contribution of antenna and core fucoses to the retention is minor when a HILIC stationary phase (e.g., an amide column) is used because both types of fucosylation have a similar positive contribution to the retention $[71,128]$. Notably, in glycopeptides also an effect in reversed-phase retention is observed when a core fucose is present. This effect again depends on the linkage of the fucose: when the fucose is $\alpha 1,6$-linked, it has a positive contribution to the retention and when the fucose is $\alpha 1,3$-linked, it has a negative contribution to the retention [132].

\section{Toward full resolution of glycan isomers}

As can be seen from Table 1, the ability to separate glycans depends highly on the complexity of the sample. When the complexity of the sample is high, separation of all structures is often not obtained. However, for glycan standards containing fewer glycan species and small saccharides, separation of the different glycan species and isomers was observed.

A low peak capacity is observed in many of the methods mentioned. This clustering of peaks can be expected because in most cases the label has a major influence on the retention. In addition, the structural difference between the glycans is often small and has only a minor influence on the retention, resulting in low selectivity. For these reasons, high-resolution glycan separations are often not obtained. To spread the signals, longer run times can be used for separation. However, this will also increase the turnaround time of the method. In addition, resolution can be increased by use of ultra-highperformance LC, where separation is performed at pressures above 400 bar $[83,133]$. Another option to obtain full separation of glycans is the addition of a second-dimension separation, as mentioned before [38, 45, 47]. A protocol for the separation of AB-labeled glycans with HILIC separation in the first dimension and reversed-phase separation in the second dimension has been described [38]. An advantage of this approach is that with these two HPLC dimensions, isobaric and isomeric structures can often be separated, which is beneficial for the characterization of individual glycans.

Notably, reversed-phase chromatography is sometimes used to desalt the sample or to create a rough separation of highly complex samples and not to obtain fully separated glycans $[49,110,113,121,134]$. This full separation of the various glycans is often not necessary when MS detection is used, because ions of a specific $\mathrm{m} / \mathrm{z}$ can be selected for further 
analysis or to obtain an extracted ion chromatogram. However, the separation of isomeric and isobaric species is of particular relevance for glycan analysis. With use of ANTS, a $(\mathrm{Glc})_{3210}$ maltooligosaccharide ladder was successfully separated by Gennaro et al. [104]. In addition, high-mannose $N$ glycan structures $(\mathrm{GlcNAc})_{2}(\mathrm{Man})_{5-9}$ from ribonuclease B and three isomers of $\mathrm{Man}_{7}$ and two isomers of $\mathrm{Man}_{8}$ were separated. However, the separation of the high-mannose structures was only visible with MS detection, because the different high-mannose species $\left(\mathrm{Man}_{5-9}\right)$ are eluted with an overlap within $4 \mathrm{~min}$. Unfortunately, in UV or fluorescence detection a distinction between overlapping peaks cannot be made. Full separation of glycan species and isomers is thus desired, which can be a challenge for complex samples containing many glycan species.

Other stationary phases might also be of use for the separation of glycan isomers. Similarly to reversed-phase chromatography, PGC chromatography is based on hydrophobic interactions with the analytes. However, in PGC chromatography polar and ionic interactions are also involved in the retention [34, 37]. It should be noted that the exact retention mechanisms of PGC chromatography are debatable [127]. The polar and ionic interactions with the analytes mean that native glycans can be separated by PGC chromatography [131]. This separation can be performed on the basis of the branching, sequence, and linkage of the glycans and therefore PGC chromatography is highly suitable for the separation of isomers [13, 135]. For example, ThaysenAndersen et al. [127] reported on the linkage-specific retention of $\alpha 2,3$ - and $\alpha 2,6$-linked sialylated glycans, showing later retention of $\alpha 2,3$-linked sialylated diantennary and triantennary glycans. Nevertheless, the robustness and reproducibility of PGC chromatography is limited [61]. PGC chromatography and HILIC have been found to be more powerful in separating glycan structural isomers than reversed-phase chromatography of the AB-labeled variants [127]. However, reversed-phase separation of $\mathrm{N}$-glycans is generally more efficient than HILIC separation, as was discussed by Walker et al. [136]. The different stationary phases might be used for different purposes, as was mentioned by Melmer et al. [61]. HILIC was described as a useful method for the analysis of complex samples, because of its high peak capacity and PGC chromatography was described as a useful method for separation of isomers. Reversed-phase chromatography was described as a useful method for quality control purposes, because of its reproducibility.

\section{Conclusion}

Reversed-phase chromatography is an technique often used for the separation of glycans, and consequently a wide variety of methods have been described. Many different columns and eluent mixtures have been used, which results in variation in separation efficiency. In addition, various labeling reagents have been used to increase hydrophobicity and detectability of the glycans. It was found that the hydrophobicity of the tag has a major influence on the separation and elution order of the different glycans. Moreover, this hydrophobicity also influences the contribution of the glycan itself to the retention.

Because of the relatively low hydrophobicity of PA, more structural information on the glycan can be derived. However, because of this low hydrophobicity, direct coupling with ESI-MS was less convenient. The more hydrophobic labels show a different elution order of glycans compared with PA-labeled glycans, but a uniform order could not be identified. Among other factors, the presence of an ion-pairing agent in the eluent, the type of column, the gradient, and the composition of the eluent have a substantial influence on the elution of glycans. Moreover, the presence of sialic acids or fucoses on the glycan also has a major influence on the retention. This influence is linkage specific, and in case of the fucose the influence also depends on whether the fucose it is located on the core or on the antenna of the $N$-glycan. In addition, the influence of sialic acids on the retention of glycopeptides is contradictory to the findings in released glycans. It appears that the exact influence of these monosaccharides is still incompletely understood, and additional research should be performed on this subject.

The quality of separation of the glycans is highly dependent on the complexity of the sample. In addition, a clustering of peaks is caused by the major contribution of the label to the retention, resulting in a low peak capacity. In complex samples, overlap between peaks of different glycan species has been observed. In fluorescence and UV-detection this causes difficulties with the identification of glycans, but for MS analysis this was often not a problem, as long as isomeric and isobaric species were separated from each other. The choice of the column, run time, and eluents influences the ability to separate isomers. In less complex samples, isomers were separated by reversed-phase chromatography. Unfortunately, no information on the elution order of these isomers was provided, which could have given insights into the retention of glycans on a reversed-phase stationary phase. The separation of isomers in complex samples could also be obtained by reversed-phase chromatography in a second dimension after HILIC separation. In addition, PGC chromatography, an emerging method for glycan separation, also has the capability to separate isomeric and isobaric glycans, also without derivatization. HILIC and PGC chromatography were compared with reversed-phase chromatography and from the data obtained it was concluded that both were better able to separate glycan species and their isomers. However, these methods have been proven to be less robust and reproducible than reversed-phase methods. Improvement of these properties might eliminate the need for reversed-phase glycan analyses in the future. 
Further research should provide more information on the influence of the sialic acids and fucoses on the retention of the glycans and also of the glycopeptides. Especially the linkage and structure specificity of the fucoses should be investigated. In addition, the analysis of samples containing triantennary and tetraantennary glycans could give more insights into the influence of the sialic acid moieties.

Acknowledgement The authors acknowledge support by the European Union (Seventh Framework Programme HighGlycan project, grant number 278535).

\section{Compliance with ethical standards}

Conflict of interest The authors declare that they have no conflict of interest.

Open Access This article is distributed under the terms of the Creative Commons Attribution 4.0 International License (http:// creativecommons.org/licenses/by/4.0/), which permits unrestricted use, distribution, and reproduction in any medium, provided you give appropriate credit to the original author(s) and the source, provide a link to the Creative Commons license, and indicate if changes were made.

\section{References}

1. Varki A, Esko JD, Colley KJ. Cellular organization of glycosylation. In: Varki A, Cummings R, Esko J, Freeze HH, Goldsmith HW, Bertozzi CR, et al., editors. Essentials of glycobiology. 2nd ed. Cold Spring Harbor: Cold Spring Harbor Laboratory Press; 2009.

2. Bertozzi C, Rabuka D, et al. Structural basis of glycan diversity. In: Varki A, Cummings R, Esko J, Freeze HH, Goldsmith HW, Bertozzi CR, editors. Essentials of glycobiology. 2nd ed. New York: Cold Spring Harbor Laboratory Press; 2009.

3. Geyer H, Geyer R. Strategies for analysis of glycoprotein glycosylation. Biochim Biophys Acta. 1764;2006:1853-69.

4. Eklund EA, Freeze HH. Essentials of glycosylation. Semin Pediatr Neurol. 2005;12:134-43.

5. Varki A. Biological roles of oligosaccharides: all of the theories are correct. Glycobiology. 1993;3:97-130.

6. Bondt A, Selman MHJ, Deelder AM, Hazes JMW, Willemsen SP, Wuhrer M, et al. Association between galactosylation of immunoglobulin $\mathrm{G}$ and improvement of rheumatoid arthritis during pregnancy is independent of sialylation. J Proteome Res. 2013;12:4522-31.

7. Haselberg R, de Jong GJ, Somsen GW. CE-MS for the analysis of intact proteins 2010-2012. Electrophoresis. 2013;34:99-112.

8. Jacobs JFM, Wevers RA, Lefeber DJ, van Scherpenzeel M. Fast, robust and high-resolution glycosylation profiling of intact monoclonal IgG antibodies using nanoLC-chip-QTOF. Clin Chim Acta. 2016;461:90-7.

9. Desaire H. Glycopeptide analysis, recent developments and applications. Mol Cell Proteomics. 2013;12:893-901.

10. El Rassi Z. Carbohydrate analysis: high performance liquid chromatography and capillary electrophoresis. Amsterdam: Elsevier; 1994.

11. Wuhrer M, Catalina MI, Deelder AM, Hokke CH. Glycoproteomics based on tandem mass spectrometry of glycopeptides. J Chromatogr B. 2007;849:115-28.

12. Kolli V, Schumacher KN, Dodds ED. Engaging challenges in glycoproteomics: recent advances in MS-based glycopeptide analysis. Bioanalysis. 2015;7:113-31.
13. Pabst M, Altmann F. Glycan analysis by modern instrumental methods. Proteomics. 2011;11:631-43.

14. Tharmalingam T, Adamczyk B, Doherty MA, Royle L, Rudd PM. Strategies for the profiling, characterisation and detailed structural analysis of N-linked oligosaccharides. Glycoconj J. 2013;30:137-46.

15. Domann PJ, Pardos-Pardos AC, Fernandes DL, Spencer DIR, Radcliffe CM, Royle L, et al. Separation-based glycoprofiling approaches using fluorescent labels. Proteomics. 2007;7 Suppl 1:70-6.

16. Dalpathado DS, Desaire H. Glycopeptide analysis by mass spectrometry. Analyst. 2008;133:731-8.

17. Merry T, Astrautsova S. Chemical and enzymatic release of glycans from glycoproteins. Methods Mol Biol. 2003;213:27-40.

18. Tarentino AL, Gómez CM, Plummer TH. Deglycosylation of asparagine-linked glycans by peptide: N-glycosidase F. Biochemistry. 1985;24:4665-71.

19. Lingg N, Zhang P, Song Z, Bardor M. The sweet tooth of biopharmaceuticals: importance of recombinant protein glycosylation analysis. Biotechnol J. 2012;7:1462-72.

20. Bereman MS, Williams TI, Muddiman DC. Development of a nanoLC LTQ Orbitrap mass spectrometric method for profiling glycans derived from plasma from healthy, benign tumor control, and epithelial ovarian cancer patients. Anal Chem. 2009;81:1130-6.

21. Ruhaak LR, Miyamoto S, Kelly K, Lebrilla CB. N-Glycan profiling of dried blood spots. Anal Chem. 2012;84:396-402.

22. Selman MHJ, Hemayatkar M, Deelder AM, Wuhrer M. Cotton HILIC SPE microtips for microscale purification and enrichment of glycans and glycopeptides. Anal Chem. 2011;83:2492-9.

23. An HJ, Peavy TR, Hedrick JL, Lebrilla CB. Determination of Nglycosylation sites and site heterogeneity in glycoproteins. Anal Chem. 2003;75:5628-37.

24. Morelle W, Michalski J-C. Analysis of protein glycosylation by mass spectrometry. Nat Protoc. 2007;2:1585-602.

25. Clark GF, Grassi P, Pang P-C, Panico M, Lafrenz D, Drobnis EZ, et al. Tumor biomarker glycoproteins in the seminal plasma of healthy human males are endogenous ligands for DC-SIGN. Mol Cell Proteomics. 2012;11:1-12.

26. Cao L, Zhang Y, Chen L, Shen A, Zhang X, Ren S, et al. Sample preparation for mass spectrometric analysis of human serum $\mathrm{N}$ glycans using hydrophilic interaction chromatography-based solid phase extraction. Analyst. 2014;139:4538-46.

27. Zhang W, Han H, Bai H, Tong W, Zhang Y, Ying W, et al. A highly efficient and visualized method for glycan enrichment by self-assembling pyrene derivative functionalized free graphene oxide. Anal Chem. 2013;85:2703-9.

28. Sun N, Deng C, Li Y, Zhang X. Highly selective enrichment of Nlinked glycan by carbon-functionalized ordered graphene/ mesoporous silica composites. Anal Chem. 2014;86:2246-50.

29. Ruhaak LR, Zauner G, Huhn C, Bruggink C, Deelder AM, Wuhrer M. Glycan labeling strategies and their use in identification and quantification. Anal Bioanal Chem. 2010;397:3457-81.

30. Rohrer JS, Thayer J, Weitzhandler M, Avdalovic N. Analysis of the $\mathrm{N}$-acetylneuraminic acid and $\mathrm{N}$-glycolylneuraminic acid contents of glycoproteins by high-pH anion-exchange chromatography with pulsed amperometric detection (HPAEC/PAD). Glycobiology. 1998;8:35-43.

31. Alpert AJ. Hydrophilic-interaction chromatography for the separation of peptides, nucleic acids and other polar compounds. J Chromatogr. 1990;499:177-96.

32. Anumula KR. Advances in fluorescence derivatization methods for high-performance liquid chromatographic analysis of glycoprotein carbohydrates. Anal Biochem. 2006;350:1-23.

33. Wuhrer M, De Boer AR, Deelder AM. Structural glycomics using hydrophilic interaction chromatography (HILIC) with mass spectrometry. Mass Spectrom Rev. 2009;28:192-206. 
34. Ruhaak LR, Deelder AM, Wuhrer M. Oligosaccharide analysis by graphitized carbon liquid chromatography-mass spectrometry. Anal Bioanal Chem. 2009;394:163-74.

35. Stavenhagen K, Kolarich D, Wuhrer M. Clinical glycomics employing graphitized carbon liquid chromatography-mass spectrometry. Chromatographia. 2014;78:307-20.

36. Kolarich D, Windwarder M, Alagesan K, Altmann F. Isomer-specific analysis of released $\mathrm{N}$-glycans by LC-ESI MS/MS with porous graphitized carbon. Methods Mol Biol. 2015;1321:427-35.

37. Fan JQ, Kondo A, Kato I, Lee YC. High-performance liquid chromatography of glycopeptides and oligosaccharides on graphitized carbon columns. Anal Biochem. 1994;219:224-9.

38. Wuhrer M, Koeleman CAM, Deelder AM. Two-dimensional HPLC separation with reverse-phase-nano-LC-MS/MS for the characterization of glycan pools after labeling with 2aminobenzamide. In: Packer NH, Karlsson NG, editors. Glycomics. New York: Humana; 2009. p. 79-91.

39. Li M, Kinzer JA. Structural analysis of oligosaccharides by a combination of electrospray mass spectrometry and bromine isotope tagging of reducing-end sugars with 2-amino-5-bromopyridine. Rapid Commun Mass Spectrom. 2003;17:1462-6.

40. Lattova E, Perreault H. Labelling saccharides with phenylhydrazine for electrospray and matrix-assisted laser desorption-ionization mass spectrometry. J Chromatogr B. 2003;793:167-79.

41. Snyder LR, Kirkland JJ. The column. In: Dolan JW, editor. Introduction to modern liquid chromatography. 3rd ed. Hoboken: Wiley; 2010. p. 199-252.

42. Claessens H, van Straten M, Cramers C, Jezierska M, Buszewski B. Comparative study of test methods for reversed-phase columns for high-performance liquid chromatography. J Chromatogr A. 1998:826:135-56.

43. $\mathrm{Hu}$ Y, Mechref Y. Comparing MALDI-MS, RP-LC-MALDI-MS and RP-LC-ESI-MS glycomic profiles of permethylated Nglycans derived from model glycoproteins and human blood serum. Electrophoresis. 2012;33:1768-77.

44. Ritamo I, Räbinä J, Natunen S, Valmu L. Nanoscale reversedphase liquid chromatography-mass spectrometry of permethylated N-glycans. Anal Bioanal Chem. 2013;405:2469-80.

45. Wuhrer M, Koeleman CAM, Fitzpatrick JM, Hoffmann KF, Deelder AM, Hokke CH. Gender-specific expression of complex-type N-glycans in schistosomes. Glycobiology. 2006;16:991-1006.

46. Robijn MLM, Koeleman CAM, Hokke CH, Deelder AM. Schistosoma mansoni eggs excrete specific free oligosaccharides that are detectable in the urine of the human host. Mol Biochem Parasitol. 2007;151:162-72.

47. Wuhrer M, Koeleman CAM, Deelder AM, Hokke CH. Repeats of LacdiNAc and fucosylated LacdiNAc on N-glycans of the human parasite Schistosoma mansoni. FEBS J. 2006;273:347-61.

48. Alley WR, Madera M, Mechref Y, Novotny MV. Chip-based reversed-phase liquid chromatography-mass spectrometry of permethylated N-linked glycans: a potential methodology for cancer-biomarker discovery. Anal Chem. 2010;82:5095-106.

49. Desantos-Garcia JL, Khalil SI, Hussein A, Hu Y, Mechref Y. Enhanced sensitivity of LC-MS analysis of permethylated Nglycans through online purification. Electrophoresis. 2011;32: 3516-25.

50. Zhou S, Hu Y, Mechref Y. High-temperature LC-MS/MS of permethylated glycans derived from glycoproteins. Electrophoresis. 2016;37:1506-13.

51. Dong X, Zhou S, Mechref Y. LC-MS/MS analysis of permethylated free oligosaccharides and $\mathrm{N}$-glycans derived from human, bovine, and goat milk samples. Electrophoresis. 2016;37:1532-48.

52. Hu Y, Shihab T, Zhou S, Wooding K, Mechref Y. LC-MS/MS of permethylated N-glycans derived from model and human blood serum glycoproteins. Electrophoresis. 2016;37:1498-505.
53. Kirsch S, Bindila L. Nano-LC and HPLC-chip-ESI-MS: an emerging technique for glycobioanalysis. Bioanalysis. 2009;1: 1307-27.

54. Hua S, Lebrilla C, An HJ. Application of nano-LC-based glycomics towards biomarker discovery. Bioanalysis. 2011;3:2573-85.

55. Meiring HD, Van Der Heeft E, Ten Hove GJ, De Jong APJM. Nanoscale LC-MS ${ }^{\mathrm{n}}$ : technical design and applications to peptide and protein analysis. J Sep Sci. 2002;25:557-68.

56. Hernández-Borges J, Aturki Z, Rocco A, Fanali S. Recent applications in nanoliquid chromatography. J Sep Sci. 2007;30:1589-610.

57. Wuhrer M, Deelder AM, Hokke CH. Protein glycosylation analysis by liquid chromatography-mass spectrometry. J Chromatogr B. 2005;825:124-33.

58. Wuhrer M, Koeleman CAM, Hokke CH, Deelder AM. Nanoscale liquid chromatography-mass spectrometry of 2aminobenzamide- labeled oligosaccharides at low femtomole sensitivity. Int J Mass Spectrom. 2004;232:51-7.

59. Schmid D, Behnke B, Metzger J, Kuhn R. Nano-HPLC-mass spectrometry and MEKC for the analysis of oligosaccharides from human milk. Biomed Chromatogr. 2002;16:151-6.

60. Royle L, Mattu TS, Hart E, Langridge JI, Merry AH, Murphy N, et al. An analytical and structural database provides a strategy for sequencing O-glycans from microgram quantities of glycoproteins. Anal Biochem. 2002;304:70-90.

61. Melmer M, Stangler T, Premstaller A, Lindner W. Comparison of hydrophilic-interaction, reversed-phase and porous graphitic carbon chromatography for glycan analysis. J Chromatogr A. 2011;1218:118-23.

62. Lareau NM, May JC, McLean JA. Non-derivatized glycan analysis by reverse phase liquid chromatography and ion mobility-mass spectrometry. Analyst. 2015;140:3335-8.

63. Williams DK, Meadows CW, Bori ID, Hawkridge AM, Comins DL, Muddiman DC. Synthesis, characterization, and application of iodoacetamide derivatives utilized for the ALiPHAT strategy. J Am Chem Soc. 2008;130:2122-3.

64. Shuford CM, Muddiman DC. Capitalizing on the hydrophobic bias of electrospray ionization through chemical modification in mass spectrometry-based proteomics. Expert Rev Proteomics. 2011;8:317-23.

65. Fenn JB. Ion formation from charged droplets: roles of geometry, energy, and time. J Am Soc Mass Spectrom. 1993;4:524-35.

66. Null AP, Benson LM, Muddiman DC. Enzymatic strategies for the characterization of nucleic acids by electrospray ionization mass spectrometry. Rapid Commun Mass Spectrom. 2003;17:2699-706.

67. Bleicher K, Bayer E. Various factors influencing the signal intensity of oligonucleotides in electrospray mass-spectrometry. Biol Mass Spectrom. 1994;23:320-2.

68. Muddiman DC, Cheng X, Udseth HR, Smith RD. Charge-state reduction with improved signal intensity of oligonucleotides in electrospray ionization mass spectrometry. J Am Soc Mass Spectrom. 1996;7:697-706.

69. Shubhakar A, Reiding KR, Gardner RA, Spencer DIR, Fernandes DL, Wuhrer M. High-throughput analysis and automation for glycomics studies. Chromatographia. 2015;78:321-33.

70. Pabst M, Kolarich D, Pöltl G, Dalik T, Lubec G, Hofinger A, et al. Comparison of fluorescent labels for oligosaccharides and introduction of a new postlabeling purification method. Anal Biochem. 2009;384:263-73.

71. Nwosu C, Yau HK, Becht S. Assignment of core versus antenna fucosylation types in protein $\mathrm{N}$-glycosylation via procainamide labeling and tandem mass spectrometry. Anal Chem. 2015;87:5905-13.

72. Albanese J, Lee R. A unique workflow for glycoprotein characterization - from sample preparation to MS/MS spectral interpretation. J Biomol Tech. 2011;22(Suppl):S57. 
73. Cook KS, Bullock K, Sullivan T. Development and qualification of an antibody rapid deglycosylation method. Biologicals. 2012;40:109-17.

74. Afiuni-Zadeh S, Rogers JC, Snovida SI, Bomgarden RD, Griffin TJ. AminoxyTMT: a novel multi-functional reagent for characterization of protein carbonylation. Biotechniques. 2016;60:186-96.

75. Zhou S, Hu Y, Veillon L, Snovida SI, Rogers JC, Saba J, et al. Quantitative LC-MS/MS glycomic analysis of biological samples using aminoxyTMT. Anal Chem. 2016;88:7515-22.

76. Harvey DJ. Electrospray mass spectrometry and fragmentation of $\mathrm{N}$-linked carbohydrates derivatized at the reducing terminus. J Am Soc Mass Spectrom. 2000;11:900-15.

77. Ruhaak LR, Steenvoorden E, Koeleman CAM, Deelder AM, Wuhrer M. 2-Picoline-borane: a non-toxic reducing agent for oligosaccharide labeling by reductive amination. Proteomics. 2010;10:2330-6.

78. Lamari FN, Kuhn R, Karamanos NK. Derivatization of carbohydrates for chromatographic, electrophoretic and mass spectrometric structure analysis. J Chromatogr B. 2003;793:15-36.

79. Anumula KR. Single tag for total carbohydrate analysis. Anal Biochem. 2014;457:31-7.

80. Chen X, Flynn GC. Analysis of N-glycans from recombinant immunoglobulin $\mathrm{G}$ by on-line reversed-phase high-performance liquid chromatography/mass spectrometry. Anal Biochem. 2007;370:147-61.

81. Chen X, Flynn GC. Gas-phase oligosaccharide nonreducing end (GONE) sequencing and structural analysis by reversed phase HPLC/mass spectrometry with polarity switching. J Am Soc Mass Spectrom. 2009;20:1821-33.

82. Adamczyk B, Tharmalingam-Jaikaran T, Schomberg M, Szekrényes Á, Kelly RM, Karlsson NG, et al. Comparison of separation techniques for the elucidation of $\mathrm{IgG} \mathrm{N}$-glycans pooled from healthy mammalian species. Carbohydr Res. 2014;389:174-85.

83. Prater BD, Connelly HM, Qin Q, Cockrill SL. High-throughput immunoglobulin G N-glycan characterization using rapid resolution reverse-phase chromatography tandem mass spectrometry. Anal Biochem. 2009;385:69-79.

84. Garner B, Merry AH, Royle L, Harvey DJ, Rudd PM, Thillet J. Structural elucidation of the $\mathrm{N}$ - and O-glycans of human apolipoprotein(a). Role of O-glycans in conferring protease resistance. J Biol Chem. 2001;276:22200-8.

85. Mariño K, Lane JA, Abrahams JL, Struwe WB, Harvey DJ, Marotta M, et al. Method for milk oligosaccharide profiling by 2-aminobenzamide labeling and hydrophilic interaction chromatography. Glycobiology. 2011;21:1317-30.

86. Mariño K, Bones J, Kattla JJ, Rudd PM. A systematic approach to protein glycosylation analysis: a path through the maze. Nat Chem Biol. 2010;6:713-23.

87. Bigge J, Patel T, Bruce J. Nonselective and efficient fluorescent labeling of glycans using 2-amino benzamide and anthranilic acid. Anal Biochem. 1995;230:229-38.

88. Gillmeister MP, Tomiya N, Jacobia SJ, Lee YC, Gorfien SF, Betenbaugh MJ. An HPLC-MALDI MS method for N-glycan analyses using smaller size samples: application to monitor glycan modulation by medium conditions. Glycoconj J. 2009;26:1135-49.

89. Kita Y, Miura Y, Furukawa J, Nakano M, Shinohara Y, Ohno M, et al. Quantitative glycomics of human whole serum glycoproteins based on the standardized protocol for liberating N-glycans. Mol Cell Proteomics. 2007;6:1437-45.

90. Hase S, Sugimoto T, Takemoto H, Ikenaka T, Schmid K. The structure of sugar chains of Japanese quail ovomucoid. The occurrence of oligosaccharides not expected from the classical biosynthetic pathway for N-glycans; a method for the assessment of the structure of glycans present in picomolar amounts. J Biochem. 1986;99:1725-33.
91. Hase S, Koyama S, Daiyasu H, Takemoto H, Hara S, Kobayashi Y, et al. Structure of a sugar chain of a protease inhibitor isolated from barbados pride (Caesalpinia pulcherrima Sw.) seeds. J Biochem. 1986;100:1-10.

92. Hase S, Ikenaka T, Matsushima Y. A highly sensitive method for analyses of sugar moieties of glycoproteins by fluorescence labeling. J Biochem. 1981;90:407-14.

93. Yanagida $\mathrm{K}$, Ogawa $\mathrm{H}$, Omichi $\mathrm{K}$, Hase S. Introduction of a new scale into reversed-phase high-performance liquid chromatography of pyridylamino sugar chains for structural assignment. J Chromatogr A. 1998;800:187-98.

94. Homma H, Tozawa K, Yasui T, Itoh Y, Hayashi Y, Kohri K. Abnormal glycosylation of serum IgG in patients with IgA nephropathy. Clin Exp Nephrol. 2006;10:180-5.

95. Hase S, Ikenaka T. Estimation of elution times on reverse-phase high-performance liquid chromatography of pyridylamino derivatives of sugar chains from glycoproteins. Anal Biochem. 1990;184:135-8

96. Hirabayashi J. Glycan profiling. In: Taniguchi N, Suzuki A, Ito Y, Narimatsu H, Kawasaki T, Hase S, editors. Experimental glycoscience. Glycochemistry. Tokyo: Springer; 2008. p. 58.

97. Chen DL, McLaughlin LW. Use of $\mathrm{pK}_{\mathrm{a}}$ differences to enhance the formation of base triplets involving C-G and G-C base pairs. J Org Chem. 2000;65:7468-74.

98. Testa AC, Wild UP. Inversion of close-lying 1.pi.* and 1.pi.pi.* states in 2-aminopyridine by protonation. A CNDO study. J Phys Chem. 1981;85:2637-9.

99. Kratschmar D, Wallner S, Florenski M, Schmid D, Kuhn R. Analysis of oligosaccharides by MEKC with aminobenzoic alkyl esters as derivatization agents. Chromatographia. 1999;50:596-600.

100. Okafo G, Langridge J, North S, Organ A, West A, Morris M, et al. High-performance liquid chromatographic analysis of complex Nlinked glycans derivatized with 2-aminoacridone. Anal Chem. 1997;69:4985-93.

101. Volpi N, Galeotti F, Yang B, Linhardt RJ. Analysis of glycosaminoglycan-derived, precolumn, 2-aminoacridone-labeled disaccharides with LC-fluorescence and LC-MS detection. Nat Protoc. 2014;9:541-58.

102. Deakin JA, Lyon M. A simplified and sensitive fluorescent method for disaccharide analysis of both heparan sulfate and chondroitin/dermatan sulfates from biological samples. Glycobiology. 2008;18:483-91.

103. Charlwood J, Birrell H, Gribble A, Burdes V, Tolson D, Camilleri P. A probe for the versatile analysis and characterization of $\mathrm{N}$ linked oligosaccharides. Anal Chem. 2000;72:1453-61.

104. Gennaro LA, Harvey DJ, Vouros P. Reversed-phase ion-pairing liquid chromatography/ion trap mass spectrometry for the analysis of negatively charged, derivatized glycans. Rapid Commun Mass Spectrom. 2003;17:1528-34.

105. Yoshinaka Y, Ueda Y, Suzuki S. Ion-pair chromatographic separation of glycoprotein derived oligosaccharides as their 8aminopyrene-1,3,6-trisulfonic acid derivatives. J Chromatogr A. 2007;1143:83-7.

106. Gennaro LA, Delaney J, Vouros P, Harvey DJ, Domon B. Capillary electrophoresis/electrospray ion trap mass spectrometry for the analysis of negatively charged derivatized and underivatized glycans. Rapid Commun Mass Spectrom. 2002;16:192-200.

107. Mason KE, Meikle PJ, Hopwood JJ, Fuller M. Characterization of sulfated oligosaccharides in mucopolysaccharidosis type IIIA by electrospray ionization mass spectrometry. Anal Chem. 2006;78: 4534-42.

108. Saba JA, Shen X, Jamieson JC, Perreault H. Effect of 1-phenyl-3methyl-5-pyrazolone labeling on the fragmentation behavior of asialo and sialylated $\mathrm{N}$-linked glycans under electrospray ionization conditions. Rapid Commun Mass Spectrom. 1999;13:704-11. 
109. Honda S. Separation of neutral carbohydrates by capillary electrophoresis. J Chromatogr A. 1996;720:337-51.

110. Saba JA, Shen X, Jamieson JC, Perreault H. Investigation of different combinations of derivatization, separation methods and electrospray ionization mass spectrometry for standard oligosaccharides and glycans from ovalbumin. J Mass Spectrom. 2001;36: 563-74.

111. Bardelmeijer HA, Lingeman H, de Ruiter C, Underberg WJ. Derivatization in capillary electrophoresis. J Chromatogr A. 1998;807:3-26.

112. Lattova E, Perreault H. Profiling of N-linked oligosaccharides using phenylhydrazine derivatization and mass spectrometry. $\mathrm{J}$ Chromatogr A. 2003;1016:71-8.

113. Lattová E, Varma S, Bezabeh T, Petrus L, Perreault H. Mass spectrometric profiling of $\mathrm{N}$-linked oligosaccharides and uncommon glycoform in mouse serum with head and neck tumor. J Am Soc Mass Spectrom. 2008;19:671-85.

114. Alpenfels W. A rapid and sensitive method for the determination of monosaccharides as their dansyl hydrazones by high-performance liquid chromatography. Anal Biochem 1981;157:153-7.

115. Walker SH, Budhathoki-Uprety J, Novak BM, Muddiman DC. Stable-isotope labeled hydrophobic hydrazide reagents for the relative quantification of $n$-linked glycans by electrospray ionization mass spectrometry. Anal Chem. 2011;83:6738-45.

116. Walker SH, Lilley LM, Enamorado MF, Comins DL, Muddiman DC. Hydrophobic derivatization of N-linked glycans for increased ion abundance in electrospray ionization mass spectrometry. J Am Soc Mass Spectrom. 2011;22:1309-17.

117. Walker SH, Papas BN, Comins DL, Muddiman DC. Interplay of permanent charge and hydrophobicity in the electrospray ionization of glycans. Anal Chem. 2010;82:6636-42.

118. Walker SH, Taylor AD, Muddiman DC. Individuality normalization when labeling with isotopic glycan hydrazide tags (INLIGHT): a novel glycan-relative quantification strategy. J Am Soc Mass Spectrom. 2013;24:1376-84.

119. Loziuk PL, Hecht ES, Muddiman DC. N-linked glycosite profiling and use of Skyline as a platform for characterization and relative quantification of glycans in differentiating xylem of Populus trichocarpa. Anal Bioanal Chem. 2016. doi:10.1007/s00216-016-9776-5.

120. Jensen PH, Karlsson NG, Kolarich D, Packer NH. Structural analysis of N- and O-glycans released from glycoproteins. Nat Protoc. 2012;7:1299-310.

121. Viseux N, de Hoffmann E, Domon B. Structural analysis of permethylated oligosaccharides by electrospray tandem mass spectrometry. Anal Chem. 1997;69:3193-8.

122. Delaney J, Vouros P. Liquid chromatography ion trap mass spectrometric analysis of oligosaccharides using permethylated derivatives. Rapid Commun Mass Spectrom. 2001;15:325-34.

123. Botelho JC, Atwood JA, Cheng L, Alvarez-Manilla G, York WS, Orlando R. Quantification by isobaric labeling (QUIBL) for the comparative glycomic study of O-linked glycans. Int J Mass Spectrom. 2008;278:137-42.

124. Sekiya S, Wada Y, Tanaka K. Derivatization for stabilizing sialic acids in MALDI-MS. Anal Chem. 2005;77:4962-8.

125. Morelle W, Page A, Michalski JC. Electrospray ionization ion trap mass spectrometry for structural characterization of oligosaccharides derivatized with 2-aminobenzamide. Rapid Commun Mass Spectrom. 2005;19:1145-58.

126. Higel F, Demelbauer U, Seidl A, Friess W, Sörgel F. Reversed-phase liquid-chromatographic mass spectrometric $\mathrm{N}$-glycan analysis of biopharmaceuticals. Anal Bioanal Chem. 2013;405:2481-93.

127. Thaysen-Andersen M, Larsen MR, Packer NH, Palmisano G. Structural analysis of glycoprotein sialylation - part I: pre-LCMS analytical strategies. RSC Adv. 2013;3:22706-26.

128. Tomiya N, Takahashi N. Contribution of component monosaccharides to the coordinates of neutral and sialyl pyridylaminated $\mathrm{N}$ glycans on a two-dimensional sugar map. Anal Biochem. 1998;264:204-10.

129. Wuhrer M, Stam JC, Van De Geijn FE, Koeleman CAM, Verrips CT, Dolhain RJEM, et al. Glycosylation profiling of immunoglobulin G (IgG) subclasses from human serum. Proteomics. 2007;7: 4070-81.

130. Takegawa Y, Deguchi K, Nakagawa H, Nishimura SI. Structural analysis of an N-glycan with "B1-4 bisecting branch" from human serum IgG by negative-ion MSn spectral matching and exoglycosidase digestion. Anal Chem. 2005;77:6062-8.

131. Nwosu CC, Aldredge DL, Lee H, Lerno LA, Zivkovic AM, German JB, et al. Comparison of the human and bovine milk Nglycome via high-performance microfluidic chip liquid chromatography and tandem mass spectrometry. J Proteome Res. 2012;11:2912-24.

132. Roitinger A, Leiter H, Staudacher E, Altmann F. HPLC method for the determination of Fuc to Asn-linked GlcNAc fucosyltransferases. Glycoconj J. 1998;15:89-91.

133. Prien JM, Prater BD, Qin Q, Cockrill SL. Mass spectrometric-based stable isotopic 2-aminobenzoic acid glycan mapping for rapid glycan screening of biotherapeutics. Anal Chem. 2010;82:1498-508.

134. Shen X, Perreault H. Electrospray ionization mass spectrometry of 1-phenyl-3-methyl-5-pyrazolone derivatives of neutral and $\mathrm{N}$ acetylated oligosaccharides. J Mass Spectrom. 1999;34:502-10.

135. Itoh S, Kawasaki N, Hashii N, Harazono A, Matsuishi Y, Hayakawa T, et al. N-linked oligosaccharide analysis of rat brain Thy- 1 by liquid chromatography with graphitized carbon column/ ion trap-Fourier transform ion cyclotron resonance mass spectrometry in positive and negative ion modes. J Chromatogr A. 2006;1103:296-306.

136. Walker SH, Carlisle BC, Muddiman DC. Systematic comparison of reverse phase and hydrophilic interaction liquid chromatography platforms for the analysis of $\mathrm{N}$-linked glycans. Anal Chem. 2012;84:8198-206. 